Received: $\quad 2019.02 .23$

Accepted: 2019.03 .17

Published: 2019.04 .02

\title{
Next-Generation Sequencing Analysis of mRNA Profile in Cisplatin-Resistant Gastric Cancer Cell Line SGC7901
}

Authors' Contribution: Study Design A Data Collection B Statistical Analysis C Data Interpretation D Manuscript Preparation E Literature Search F Funds Collection G

\author{
$A B 1$ Zhenwei Deng* \\ $A C 2$ Huaiming Wang* \\ CDF 3 Guohu Guo* \\ DE 1,4 Xiyao Li \\ BD 1 Yongchang Cai \\ DE 1 Yuxin Tang \\ CF 1 Yijun Wang \\ BD 1 Jiabao Li \\ BF 1 Zhibin Lu \\ $A B C 5$ Xueqiao Yu \\ DE 1 Ruiping Li \\ ABF 1 Libo Li
}

\author{
1 Department of General Surgery, Dongguan People's Hospital, Southern Medical \\ University, Dongguan, Guangdong, P.R. China \\ 2 Guangdong Provincial Key Laboratory of Colorectal and Pelvic Floor Diseases, \\ Gastrointestinal Institute of Gastroenterology, Sixth Affiliated Hospital of Sun Yat- \\ sen University, Guangzhou, Guangdong, P.R. China \\ 3 Department of General Surgery, Second Affiliated Hospital of Shantou University \\ Medical College, Shantou, Guangdong, P.R. China \\ 4 Department of General Surgery, First Hospital of China Medical University, \\ Shenyang, Liaoning, P.R. China \\ 5 Department of Colorectal and Anal Surgery, Clinical Center of Intestinal and \\ Colorectal Diseases of Hubei Province, Key Laboratory of Intestinal and Colorectal \\ Diseases of Hubei Province, Zhongnan Hospital of Wuhan University, Wuhan, \\ Hubei, P.R. China
}

Corresponding Authors: Source of support:
* Zhenwei Deng, Huaiming Wang and Guohu Guo contributed equally to this work Libo Li, e-mail: 13602842160@163.com, Ruiping Li, e-mail: Irp0919@126.com, Xueqiao Yu, e-mail: yuxueqiao2008@163.com Natural Science Foundation of Guangdong Province, China (No. 2017A030310407)

Background: Cisplatin-resistant gastric cancer (GC) occurs in patients with GC treated with cisplatin-based chemotherapy, which results in disease progression and early recurrence during the treatment.

Material/Methods: To understand the initiation and developmental mechanism underlying cisplatin-resistant GC, we developed cisplatin-resistant SGC7901 cells (SGC7901/DDP) from the parental cells (SGC7901/S) by continuous exposure to increasing concentrations of cisplatin and subjected these 2 cell lines to RNA sequencing analysis. The data were verified by quantitative polymerase chain reaction and their functional role was evaluated by cell counting kit 8 assay and cell apoptosis and cell cycle flow cytometric analysis. Bioinformatics analysis was performed to classify the differentially-expressed genes (DEGs) involved in the development of cisplatin resistance.

Results: In comparison with SGC7901/S cells, SGC7901/DDP cells showed a total of 3165 DEGs (2014 upregulated and 1151 downregulated, fold change $\geq 2$, and adjusted $P$ value $<0.001$ ). qRT-PCR confirmed the reliability of the RNA sequencing results. Depletion of the top 5 upregulated mRNAs reversed the resistant index, increased apoptotic SGC7901/DDP cells, and arrested the cells at G2/M phase. Gene ontology analysis revealed that the DEGs mainly regulate metabolic process, immune system, locomotion, cell adhesion, cell growth, cell death, cytoskeleton organization, cell binding, signal transducing activity, and antioxidant activity. Kyoto Encyclopedia of Genes and Genomes analysis showed that the DEGs were mainly involved in the PI3K-Akt signaling pathway, Rap1 signaling pathway, proteoglycans in cancer, regulation of actin cytoskeleton, and pathways in cancer.

Concluisons: The present study is the first to interrogate mRNAs profiles in human GC cells with cisplatin resistance using RNA sequencing, which may assist in discovering potential therapeutic targets for cisplatin-resistant GC patients.

MeSH Keywords: $\quad$ Cisplatin • Drug Resistance • Sequence Analysis, RNA • Stomach Neoplasms

Full-text PDF: https://www.medscimonit.com/abstract/index/idArt/915866

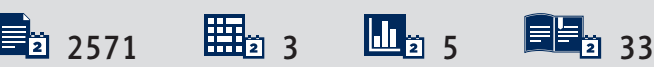




\section{Background}

Gastric cancer (GC) is one of the major malignancies in the world, especially in East Asia [1,2]. Newly diagnosed gastric cancers in China account for $44.2 \%$ of new gastric cancers globally. In China, most GC is diagnosed at an advanced stage [3]. At present, patients with GC in China are characterized by " 3 highs and 3 lows": high incidence rate, high metastasis rate, high mortality rate, low early diagnosis rate, low radical resection rate, and low survival rate. Comprehensive treatment of systemic chemotherapy is currently the main treatment method [4]. The 2016 National Clinical Cancer Network (NCCN) recommends cisplatin as a front-line treatment for advanced GC, as the median survival time and disease-free survival time in platinum-based chemotherapy groups is significantly longer than with non-cisplatin-containing regimens [5-7]. However, this preferred treatment can be dramatically compromised by tumor drug resistance [8]. Since this reduces the topical drug concentration in the cancer region, clinicians usually choose to increase the drug dosage or change the therapy scheme. Nevertheless, these alternatives can also lead to systemic toxicity, such as liver damage, bone marrow suppression, neuritis, and other unknown adverse events [9]. Consequently, there is an urgent need to explore the mechanism underlying regulation of cisplatin resistance and to identify other ways to improve cancer treatments.

Mechanisms of cisplatin resistance include enhanced DNA repair ability, high activity of Glutathione S-transferase (GSTs), spontaneous cell autophagy, and high expression of P-glycoprotein (P-gp) [10-13]. Emerging evidence has demonstrated that abnormal expression of proteins (e.g., microtubule-associated serine/threonine kinase 1, copper transporter 1), noncoding RNA (IncRNA, (e.g., IncRNA KCNQ1OT and IncRNA MEG3) or miRNAs (e.g., miR-363 and miR-2) can regulate the chemotherapy sensitivity of cancer cells to cisplatin [14-17]. Furthermore, nextgeneration sequencing technology has been widely utilized for effective target screening, especially in biological applications. The next-generation sequencing analysis is an open discovery system for discovering new genetic information, which has been applied for searching potential targets in some diseases. Various studies have searched for potential targets during cisplatin resistance [18]. However, the underlying mechanisms of cisplatin resistance are quite complex, and have not been fully elucidated.

In this study, we constructed the SGC7901/DDP (resistant to cisplatin) cells, and then analyzed mRNA expression profiles, exploring more molecular targets for chemotherapy and guiding appropriate methods for GC with cisplatin resistance.

\section{Material and Methods}

\section{Cell culture}

The SGC7901/S (sensitive to cisplatin) human GC cell line was obtained from the Cell Bank of the Chinese Academy of Sciences (Shanghai, People's Republic of China). SGC7901/S cells were exposed to cisplatin (Dalian Meilun, People's Republic of China) at an initial concentration of $0.01 \mathrm{mg} / \mathrm{L}$. Subsequently, surviving cells, which were tolerant to cisplatin, were selected and cultured in cisplatin at twice the previous concentration. This procedure was repeatedly applied to the cells until they could grow well in the presence of $2.0 \mathrm{mg} / \mathrm{L}$ cisplatin. SGC7901/DDP (resistant to cisplatin) cells were successfully established when the cells were tolerant to $2.0 \mathrm{mg} / \mathrm{L}$ cisplatin. The SGC7901/DDP cells were continuously exposed to cisplatin for maintenance of resistance. The cells were cultured in RPMI-1640 (Gibco, Carlsbad, CA with $10 \%$ fetal bovine serum (FBS, Gibco) at $37^{\circ} \mathrm{C}$ in a humidified $5 \% \mathrm{CO}_{2}$ incubator.

\section{Cell viability analysis}

Cells were seeded into 96-well plates at a density of 3000/well and cultured for $24 \mathrm{~h}$. Then, the cells were treated with different concentrations of cisplatin $(0.01,0.1,1,10,20,50$, or $100 \mu \mathrm{g} / \mathrm{mL}$ ). Cells were quantified after $48 \mathrm{~h}$ using the CCK-8 assay (Donjindo, Kumamoto, Japan) following the manufacturer's protocols. The OD value at $450 \mathrm{~nm}$ were collected for cell viability analysis from a microplate reader. The half-maximal inhibitory concentration (IC50) was determined via the OD value above, and then the resistance index (RI) was calculated with the formula: RI=IC50 (SGC7901/DDP)/IC50 (SGC7901/S).

\section{RNA sequencing analysis}

Total RNA was extracted from the cells using TRlzol reagent according to the standard protocol (Invitrogen, Carlsbad, CA). RNA concentration and purity were evaluated using a NanoDrop 2000 spectrophotometer (Thermo Fisher Scientific, Waltham, MA). RNA integrity (RIN $\geq 7$ and $28 \mathrm{~S} / 18 \mathrm{~S} \geq 0.7$ ) was assessed on an Agilent 2100 Bioanalyzer system (Agilent Technologies, Santa Clara, CA). Oligo (dT) magnetic beads (Invitrogen) were used to enrich mRNA. Following fragment screening, library building, and $P C R$ product purification, the samples were sequenced on a BGISEQ-500 platform at the BGI Bioinformatics Corporation (Shenzhen, China). After primary sequencing data passed quality control, the raw reads were filtered to obtain clean reads, which were aligned to the reference genome using HISAT and to reference gene sequences using Bowtie2. Gene data were assessed using RSEM software. The differentially expressed genes (DEGs) between SGC7901/DDP cells and SGC7901/S cells were identified using the Poisson distribution method. The significance of DEGs were determined by the threshold 
with an adjusted $P$ value [FDR $q$ value] $<0.001$ and cut-off of $\mid$ Log2Ratio $\mid \geq 1$.

\section{GO (Gene Ontology) analysis and Kyoto Encyclopedia of Genes and Genomes database (KEGG) pathway analysis}

GO analysis was carried out to elucidate the biological functions of the DEGs in the experiment. We downloaded the GO annotations from Gene Ontology (http://www.geneontology.org/). This analysis classifies the functions of these DEGs into 3 aspects: biological process, cellular component, and molecular function. Fisher's exact test was applied to identify the significant GO terms, and FDR was utilized to correct the $P$ values. Pathway analysis was utilized to find out the significant pathways of the DEGs according to KEGG. Fisher's exact test was used to select the significant pathways, and the threshold of significance was defined FDR.

\section{Quantitative real-time PCR analysis (qRT-PCR)}

Total RNA was extracted as mentioned above and reversely transcribed into cDNA using the PrimeScript RT Master Mix (TaKaRa, Dalian, China). The expression of target genes randomly selected from the top 20 dysregulated DEGs that were independently validated using the SYBR Green I PCR Kit (Takara) following the manufacturer's standard protocols. Fold change $\left(2^{-\Delta C t}\right)$ was normalized to GAPDH [19]. The primer sequences are shown in Table 1.

\section{Western blot analysis}

The protein samples were extracted using RIPA lysis buffer (Beyotime Biotechnology, Shanghai, China) supplemented with protease inhibitors. A total of $40 \mu \mathrm{g}$ protein was separated and transferred onto nitrocellulose membranes. Membranes were incubated with primary antibodies to E-cadherin (1: 1000; Cell Signaling Technology, Danvers, MA), N-cadherin (1: 1000; Cell Signaling Technology), Vimentin (1: 1000; Cell Signaling Technology) and $\beta$-actin (1: 1500; Abclonal, Wuhan, China) at $4^{\circ} \mathrm{C}$ overnight, followed by incubation with appropriate secondary antibodies. Protein bands were captured using the ChemiDocTM XRS system (Bio-Rad, Shanghai, China).

\section{RNA interference assay}

Tow specific siRNA targeting the top 5 upregulated mRNAs (CBSL, SORBS2, GAGE12B, LOC101927345, RBM14-RBM4) and negative control (NC) siRNA were bought from Ribobio Co., LTD (Guangzhou, China). SGC7901/DDP cells were suspended in Opti-MEM (Invitrogen) and reverse transfections were performed with a 1: 1 mixture of 2 siRNAs using Lipofectamine 3000 (Invitrogen) according to the manufacturer's instructions. The siRNAs sequences are indicated in Table 2.
Table 1. Primers used in quantitative real-time PCR analysis.

\begin{tabular}{|c|c|c|}
\hline Target gene & Sequence $\left(5^{\prime}-3^{\prime}\right)$ & $\begin{array}{c}\text { Product } \\
\text { length (bps) }\end{array}$ \\
\hline CBSL-F & GTGAACAATCAGCGGCATTTAG & \multirow{2}{*}{84} \\
\hline CBSL-R & GGGTGATGACAGAATGTTCCA & \\
\hline GAGE12B-F & GCTATGTACAGCCTCCTGAAAT & \multirow{2}{*}{111} \\
\hline GAGE12B-R -F & GATCCTGACGTTGAGTTGCT & \\
\hline ANK2-F & CCCAGAAACAGTCACAGAAGAA & \multirow{2}{*}{98} \\
\hline ANK2-R & CAGAGGATACATACCGCCTAATG & \\
\hline FGF22-F & ТСТТСТССТССАСТСАСТТСТ & \multirow{2}{*}{142} \\
\hline FGF22-R & CCTGAGGACACTGCTTTGAT & \\
\hline PLGLB2-F & CTAAGACTTGGCTGTCACAGTAG & \multirow{2}{*}{98} \\
\hline PLGLB2-R & CAGCATTTGTGTGGTCAGATTT & \\
\hline MSMB-F & CATGTTGCACCCTTGTTTCTAC & \multirow{2}{*}{102} \\
\hline MSMB-R & ССТTСТTСТССАССАСGATATAC & \\
\hline PLA2G4B-F & CAACCTCCAGGACAGCTTATAC & \multirow{2}{*}{128} \\
\hline PLA2G4B-R & CTGTTGAGGGTGGTTCTTCTATC & \\
\hline GSG1L2-F & TGTAGGAGTTTCCGGAGTGTA & \multirow{2}{*}{105} \\
\hline GSG1L2-R & GATGGCGCTTGTCAGTATCA & \\
\hline SERPINB3-F & CAGCAAGAGGAGAGGCATAAA & \multirow{2}{*}{121} \\
\hline SERPINB3-R & TGGTGAACTCGATGTGATCTG & \\
\hline MSMP-F & CTGCTTGGTGATGTCTCTACTC & \multirow{2}{*}{141} \\
\hline MSMP-R & GTGCAATGGAAACAGTCCTTG & \\
\hline GAPDH-F & GGTGTGAACCATGAGAAGTATGA & \multirow{2}{*}{123} \\
\hline GAPDH-R & GAGTCCTTCCACGATACCAAAG & \\
\hline
\end{tabular}

Table 2. siRNAs used in RNA interference assay.

\begin{tabular}{lc}
\multicolumn{1}{c}{ Target gene } & \multicolumn{1}{c}{ Sequence (5'-3') } \\
CBSL & \#1 CGGCAAAATCTCCAAAAATCTTG \\
& \#2 TGCCAGATATTCTGAAGAAAATC \\
SORBS2 & \#1 CAGCCATTTTACTTATGGAAAAC \\
& \#2 AGCCATTTTACTTATGGAAAACA \\
GAGE12B & \#2 TTCCTGCCGTCCGGACTCTTTTT \\
& \#1 AGCAGTTAAACAAGGATATAATA \\
LOC101927345 & \#2 CAGTTAAACAAGGATATAATACA \\
& \#1 CGGCGACAAAATGAAGATATTCG \\
RBM14-RBM4 & \#2 CTGTTCTTCTGTCCTTCAATACT \\
\hline Negative control & TTCTCCGACGTGTCACGT \\
\hline
\end{tabular}




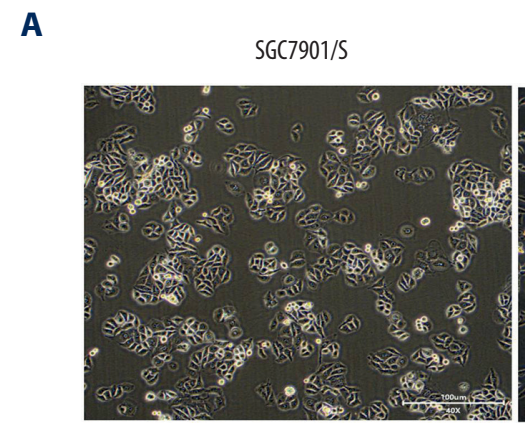

C

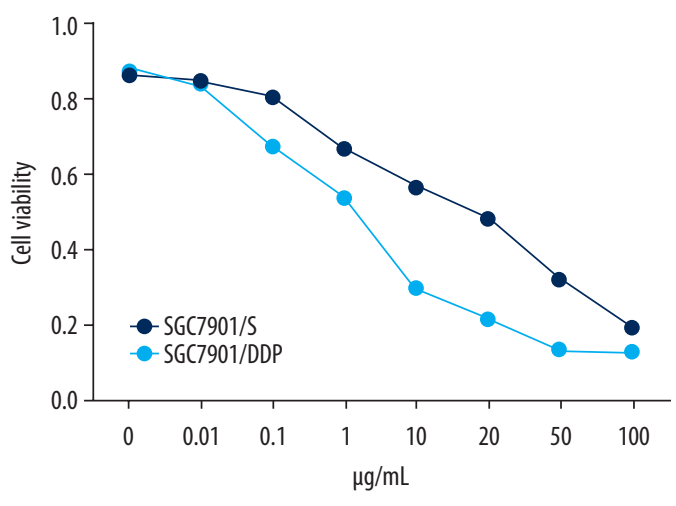

E

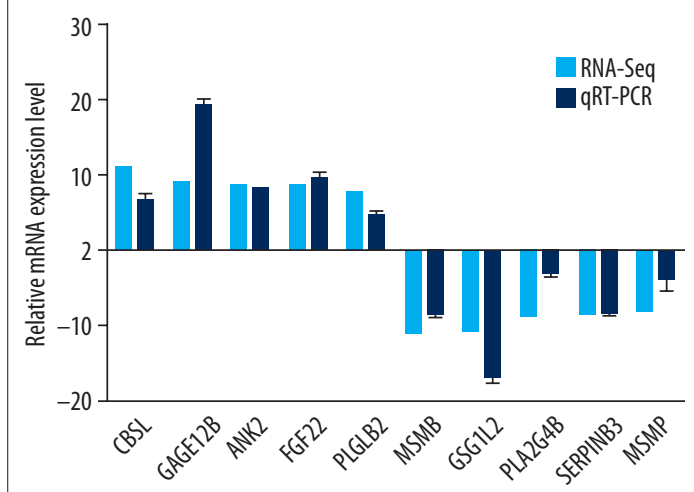

B

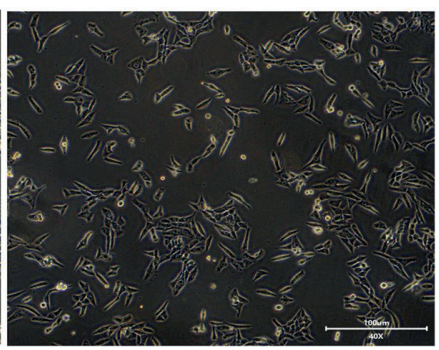

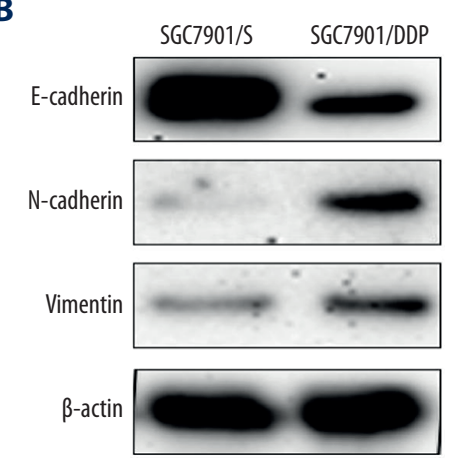

D

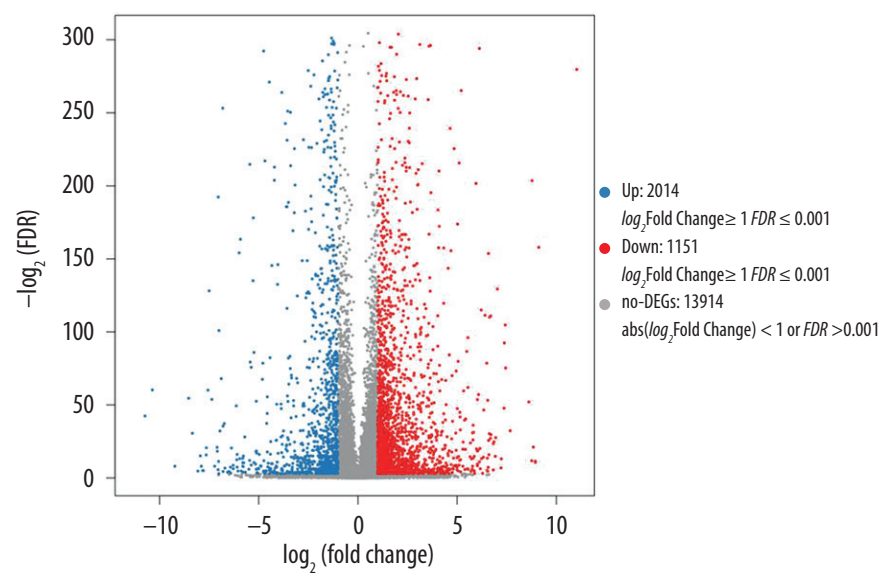

$\mathbf{F}$

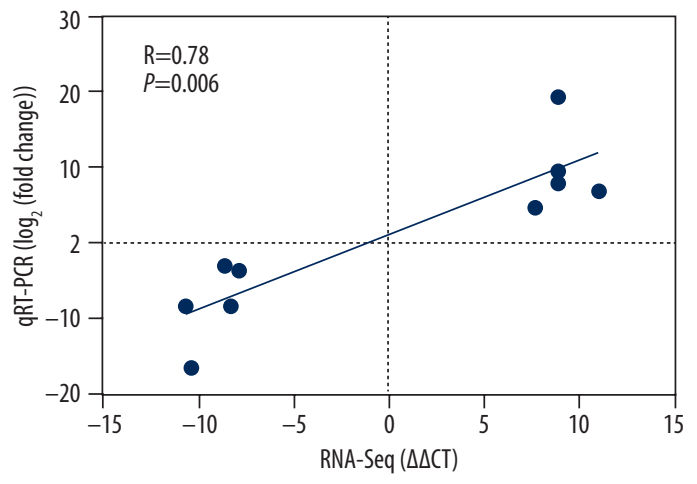

Figure 1. RNA sequencing analysis of SGC7901/DDP cells. (A) Phase-contrast micrographs of SGC7901 cells treated with $0.01 \mathrm{mg} / \mathrm{L}$ cisplatin for $24 \mathrm{~h}$. Scale bar: $100 \mu \mathrm{m}$. (B) The protein levels of epithelial-mesenchymal makers in SGC7901 cells treated with $0.01 \mathrm{mg} / \mathrm{L}$ cisplatin for $24 \mathrm{~h}$. (C) CCK8 assay for SGC7901/S cells and SGC7901/DDP cells treated with cisplatin (0, 0.01, $0.1,1,10,20,50$, and $100 \mu \mathrm{g} / \mathrm{mL}$, respectively). (D) Volcano plot for all the genes in SGC7901/DDP cells compared with SGC7901/S cells. The blue and red dots indicated the up- and downregulated DEGs (FDR q value $<0.001$ and fold change $\geq 2$ ). (E) The DEGs detected by RNA sequencing were confirmed by qRT-PCR. (F) The linear regression with Pearson's correlation coefficient showed the consistency between RNA sequencing and qRT-PCR data. 
Table 3. Top 20 dysregulated mRNAs in RNA sequence analysis.

\begin{tabular}{|c|c|c|c|c|}
\hline Gene ID & Gene symbol & Log2 fold change & FDR & Status \\
\hline 102724560 & CBSL & 11.02583167 & $1.46 \mathrm{E}-280$ & Up \\
\hline 8470 & SORBS2 & 9.118941073 & $8.58 \mathrm{E}-159$ & Up \\
\hline 729428 & GAGE12B & 8.942514505 & $1.68 \mathrm{E}-11$ & Up \\
\hline 101927345 & LOC101927345 & 8.936637939 & $2.25 \mathrm{E}-12$ & Up \\
\hline 100526737 & RBM14-RBM4 & 8.839203788 & $5.12 \mathrm{E}-22$ & Up \\
\hline 287 & ANK2 & 8.77148947 & $2.52 \mathrm{E}-204$ & Up \\
\hline 27006 & FGF22 & 8.758223215 & $1.15 \mathrm{E}-12$ & Up \\
\hline 284040 & CDRT4 & 8.614709844 & $7.06 \mathrm{E}-53$ & Up \\
\hline 5342 & PLGLB2 & 7.672425342 & $3.64 \mathrm{E}-33$ & Up \\
\hline 93649 & MYOCD & 7.442943496 & $4.54 \mathrm{E}-76$ & Up \\
\hline 6781 & STC1 & -11.3426303 & 0 & Down \\
\hline 4477 & MSMB & -10.7287708 & $3.30 \mathrm{E}-43$ & Down \\
\hline 644070 & GSG1L2 & -10.356452 & $4.32 \mathrm{E}-61$ & Down \\
\hline 84560 & MT4 & -9.22881869 & $7.75 \mathrm{E}-09$ & Down \\
\hline 100137049 & PLA2G4B & -8.53527538 & $2.52 \mathrm{E}-55$ & Down \\
\hline 6317 & SERPINB3 & -8.33985 & $2.08 \mathrm{E}-31$ & Down \\
\hline 27290 & SPINK4 & -8.06608919 & $1.46 \mathrm{E}-05$ & Down \\
\hline 8214 & DGCR6 & -7.94251451 & $4.97 \mathrm{E}-16$ & Down \\
\hline 692094 & MSMP & -7.88874325 & $7.76 \mathrm{E}-09$ & Down \\
\hline 2520 & GAST & -7.82017896 & $7.35 \mathrm{E}-06$ & Down \\
\hline
\end{tabular}

\section{Flow cytometric analysis}

Cells were transfected with siRNAs for $48 \mathrm{~h}$ and were suspended in PBS for further experiments. Cell cycle distribution and apoptotic cell proportion were analyzed on a FACSCanto II flow cytometer (BD Biosciences) and detected using a cell cycle detection kit (Keygen, Nanjing, China) and the Annexin-V-FITC/PI apoptosis detection kit (Keygen), respectively, as per the manufacturers' instructions.

\section{Statistical analysis}

Data are presented as the means \pm standard deviation (SD). Statistical evaluation of the data was performed using SPSS 22.0 software (SPSS, Chicago, IL). The comparable results from at least 3 independent experiments were statistically calculated using the unpaired $t$ test and one-way ANOVA. Linear regression was performed with Pearson's correlation coefficient (R). Differences were considered significant when $P<0.05$.

\section{Results}

\section{Expression profiles mRNAs in SGC7901/DDP cells}

We developed cisplatin-resistant SGC7901 cells (SGC7901/DDP) from the parental cells (SGC7901/S) by continuous exposure to increasing concentrations of cisplatin, which caused SGC7901 cells to undergo the transformation from epithelial to mesenchymal appearance (Figure 1A), as indicated by decreased expression of the epithelial marker E-cadherin, and increased expression of the mesenchymal markers $\mathrm{N}$-cadherin and Vimentin (Figure 1B). We verified the drug sensitivity of SGC7901/DDP cells using CCK-8 assay (Figure 1C). The results showed that the IC50 of SGC7901/DDP cells is $24.93 \pm 1.63 \mu \mathrm{g} / \mathrm{ml}$ compared with $1.948 \pm 0.373 \mu \mathrm{g} / \mathrm{ml}$ in SGC7901/DDP cells, and the RI for SGC7901/DDP cell lines was 12.80 , suggesting these cells are resistant to cisplatin. We then detected the mRNAs profile of SGC7901/DDP cells and SGC7901/S cells using the secondgeneration BGISEQ-500 RNA sequencing platform (detailed information could be accessed with GSE122130 at GEO database). We used volcano plots to assess and compare gene expression variation between the SGC7901/DDP cells and SGC7901/S cells (Figure 1D). Compared to SGC7901/S cells, a total of 2014 mRNAs exhibited upregulated expression levels 

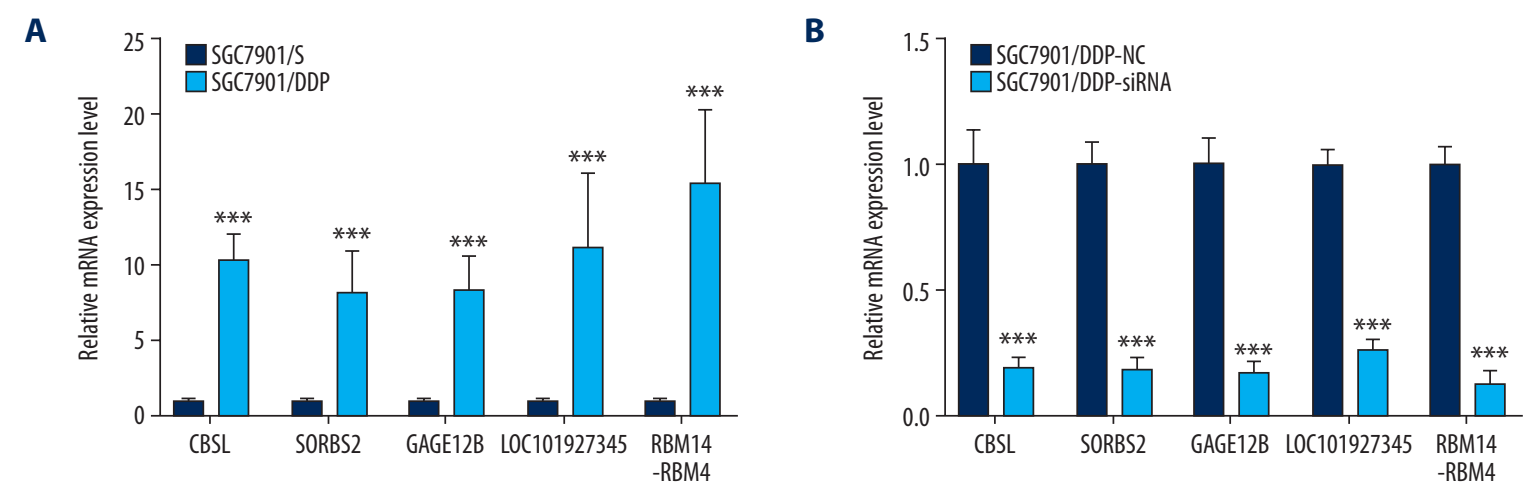

Figure 2. RNA interfering of the top 5 cisplatin-resistant related genes (CBSL, SORBS2, GAGE12B, LOC101927345, RBM14-RBM4) in SGC7901/DDP cells. (A) Verification of the top 5 cisplatin-resistant-related genes in SGC7901/DDP cells compared with SGC7901/S ones using qRT-PCR. (B) The interfering efficacy of the siRNAs was determined by qRT-PCR. ${ }^{* * *} P<0.001$, one-way ANOVA analysis; error bar: \pm SD.

and 1151 mRNAs exhibited downregulated expression levels in SGC7901/DDP cells, indicating that these mRNAs might also have common functions in facilitating the drug resistance of SGC7901 cells to cisplatin. The top 20 dysregulated mRNAs from our RNA sequencing are listed in Table 3.

\section{Verification of dysregulated mRNAs using qRT-PCR assay}

We randomly selected 5 upregulated or downregulated mRNAs (CBSL, GAGE12B, ANK2, FGF22, PLGLB2, SERPINB3, $M S M P$ ) from the top 10 dysregulated genes for qRT-PCR verification. The relative fold changes (SGC7901/DDP compared to SGC7901/S) detected by qRT-PCR were in agreement with the RNA sequencing results (Figure $1 E, 1 F$ ), indicating the reliability of our experiment data.

\section{Verification of the functional role of the upregulated mRNAs using RNAi experiments}

The top 5 mRNAs (CBSL, SORBS2, GAGE12B, LOC101927345, RBM14-RBM4) that were highly expressed in SGC7901/DDP cells compared with SGC7901/S cells were selected for further loss-of-function study (Figure 2A). We knocked down these selected mRNAs, which were confirmed by qRT-PCT assay (Figure 2B), and then performed cell function experiments to validate their anti-proliferative effect on SGC7901/DDP cells. As shown in Figure 3A, 3B, knockdown of these 5 genes attenuated the drug resistance of SGC7901/DDP cells, as well as decrease the RI, respectively. Moreover, knockdown of these 5 genes also induced cell apoptosis (Figure 4A, 4C) and arrested cell cycle at G2/M phase (Figure 4B, 4D). These results further confirmed that the dysregulated mRNAs obtained from our RNA sequencing assay contributed to the modulation of cisplatin resistance of SGC7901 cells.

\section{Bioinformatics analysis of the DGEs}

GO annotation and enrichment analysis was performed to determine which biological processes, cellular components, and molecular functions are affected by the differentially expressed mRNA in SGC7901/DDP cells. In the GO pathway enrichment analysis, the most frequently predicted functions of aberrant mRNAs were metabolic process, immune system, locomotion, cell adhesion, cell growth, cell kill, cytoskeleton organization, cell binding, signal transducing activity, and antioxidant activity (Figure 5A).

Through KEGG pathway analysis, we discovered which pathways were implicated in drug resistance property, and better defined the biological functions of significantly dysregulated mRNAs. The top 20 enrichment KEGG terms are exhibited in Figure $5 \mathrm{~B}$, indicating that the differentially expressed mRNAs are associated with the PI3K-Akt signaling pathway, Rap1 signaling pathway, proteoglycans in cancer, regulation of actin cytoskeleton, and pathways in cancer.

\section{Discussion}

Surgery remains the main therapy for GC, but recent studies proved that perioperative and postoperative adjuvant chemotherapy and chemoradiation can improve the outcome of patients with advanced GC, and cisplatin constitutes the major therapeutic option [20]. Unfortunately, cancer cells always become resistant to cisplatin-based therapies, thus leading to relapse and therapeutic failure. For this reason, exploring the molecular mechanisms of cisplatin resistance is of great significance, even if it is far from completely elucidated to date. In this study, we conducted mRNA sequencing using SGC7901/S and SGC7901/DDP cells on a BGISEQ-500 sequencer. 
A

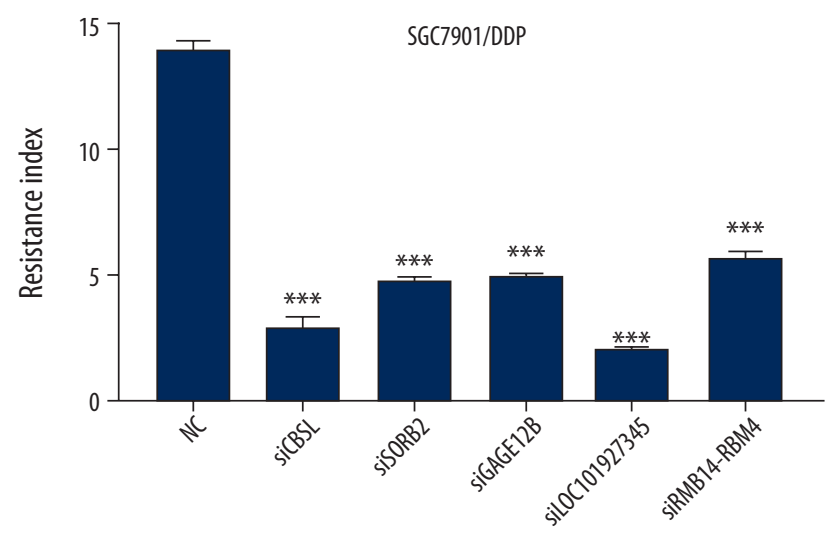

B
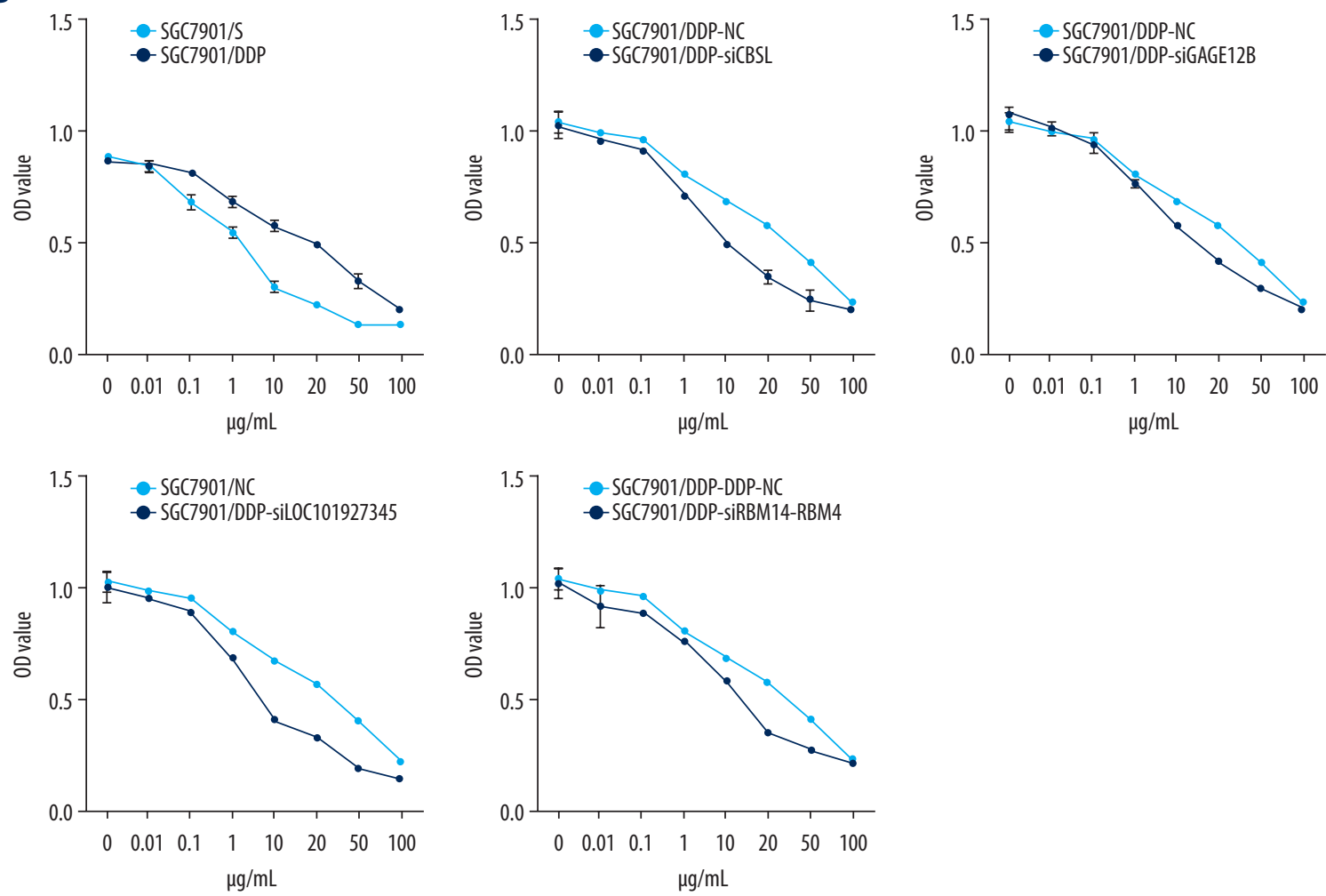

Figure 3. Drug resistance of SGC7901/DDP cells after 5 cisplatin-resistant related genes (CBSL, SORBS2, GAGE12B, LOC101927345, RBM14-RBM4) depletion. (A) Resistance index of SGC7901/DDP after RNA interfering. (B) CCK8 assay for SGC7901/DDP cells treated with cisplatin $\left(0,0.01,0.1,1,10,20,50\right.$, and $100 \mu \mathrm{g} / \mathrm{mL}$, respectively) after RNA interfering. ${ }^{* *} P<0.001$, one-way ANOVA analysis; error bar: \pm SD.

After validations of the expression and function of the uncovered differentially expressed mRNAs, GO annotation and enrichment analysis and KEGG pathway analysis were carried out to explore the potential mechanisms and chemotherapeutic molecular targets to cisplatin resistance.

The best-expounded mode of action of cisplatin are DNA damage response and mitochondrial apoptosis [21,21]. When the degree of DNA damage is limited, cisplatin adducts induce cell cycle arrest in the $S$ and $G 2$ phases, which allows the repair mechanisms to reconstruct DNA integrity and prevent abortive or abnormal mitoses [23]. Nevertheless, if the DNA damage is not repaired, cells die (mostly by apoptosis). To validate the reliability of the RNA sequencing data, siRNAs-based rescue experiments, including cell viability assay, cell cycle distribution, and apoptotic cell proportion analyses, were conducted, 


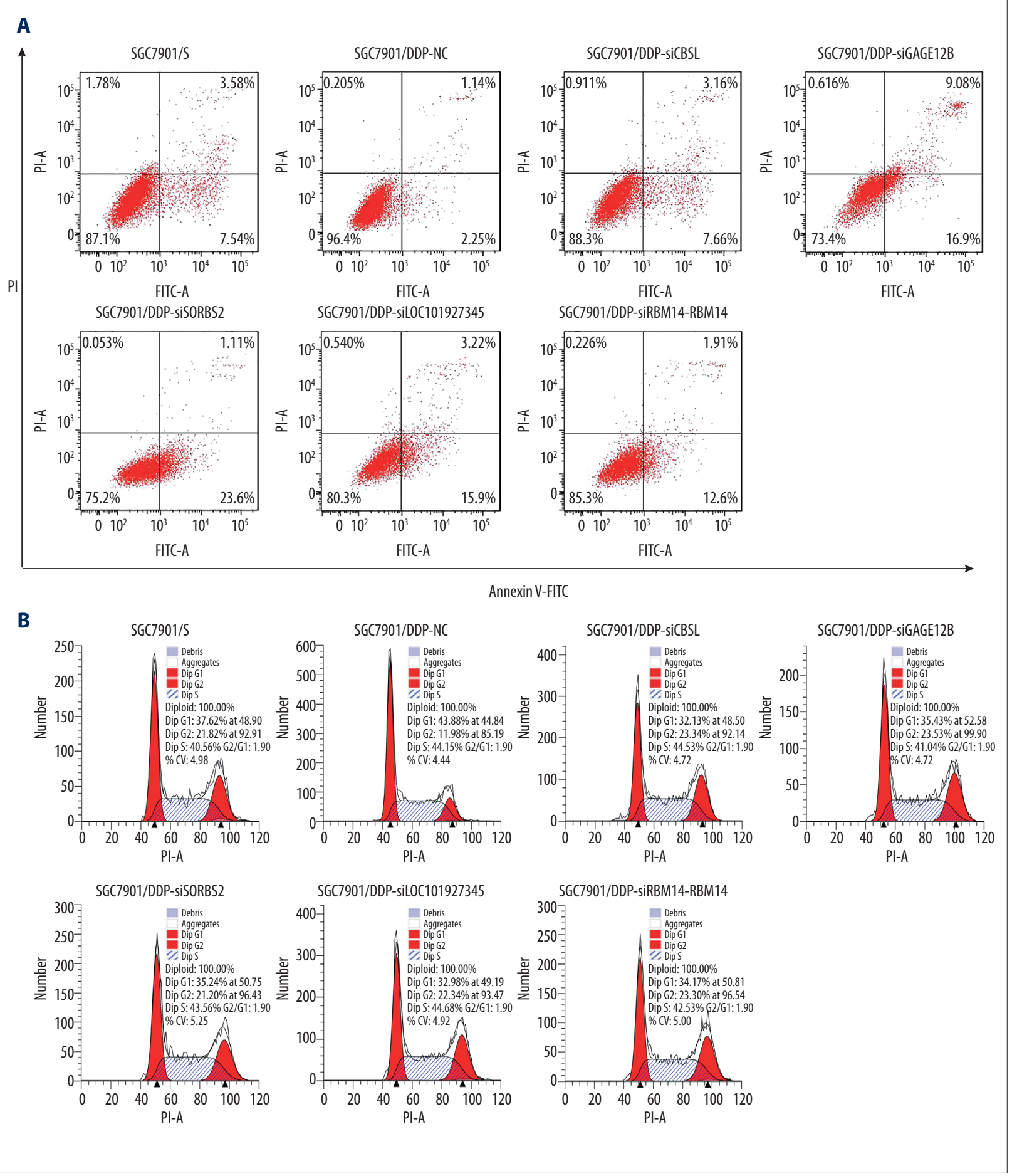



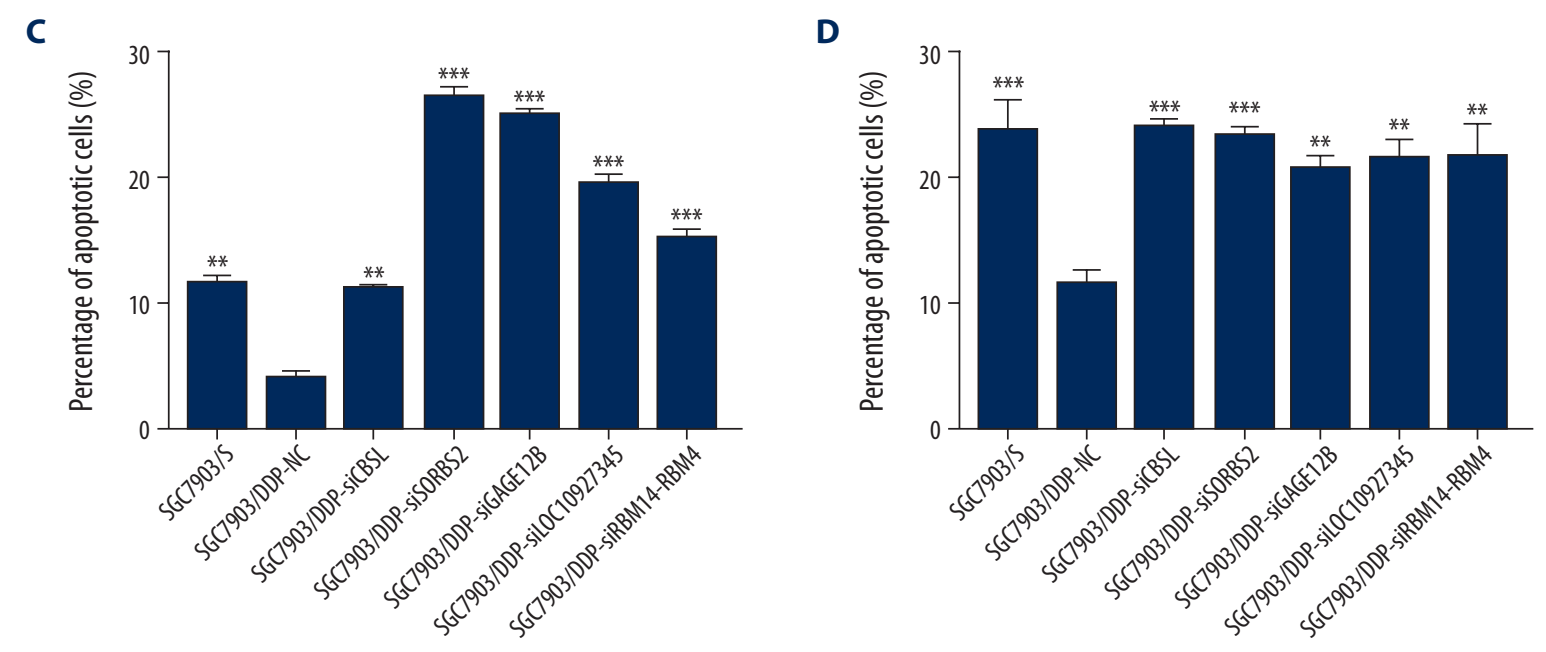

Figure 4. Flow cytometric analysis for Annexin-V staining and PI staining in SGC7901/DDP cells after 5 cisplatin-resistant genes (CBSL, SORBS2, GAGE12B, LOC101927345, RBM14-RBM4) depletion. (A, C) The cells stained with annexin-V were counted as apoptotic cells, and data are presented as the percentage of apoptotic cells. (B, D) FACS analysis showed the cell cycle distribution, and data are presented as the percentage of G2/M phase arrested cells. ${ }^{* *} P<0.01,{ }^{* * *} P<0.001$, one-way ANOVA analysis; error bar: \pm SD.

and the results displayed that after knockdown of the top 5 upregulated genes (CBSL, GAGE12B, SORBS2, LOC101927345, RBM14-RBM4) in SGC7901/DDP cells compared to SGC7901/S cells, the drug resistance were attenuated, the apoptotic rate increased, and cell cycle was arrested at G2/M phase, which indicated that they might function as boosters to cisplatin resistance. However, there were few studies on their effect, and the present study appears be the first to elucidate their function in cancer. We also noted that many genes showing differential expression in RNA sequencing data have been demonstrated to be associated with cisplatin resistance in cancers, such as ABCA8 in ovarian cancer [24], HOXD3 in breast cancer [25], and HSPB1 in nasopharyngeal carcinoma [26]. Nevertheless, our study also uncovered dozens of DEGs that were enriched in cell growth (biological process) as revealed by $\mathrm{GO}$ analysis, which not only proves the reliability of the RNA sequencing data, but also revealed numerous potential targets that regulate cisplatin resistance.

Cancer stem cells (CSCs), largely attributed to the cellular biological program termed epithelialmesenchymal transition (EMT), are more resistant than non-CSCs of the same cancers to conventional chemotherapy [27]. For instance, previous research demonstrated that CD44+ (the only CSC marker of 3 GC cell lines, AGS, MKN-45, and N87, associated with tumor formation in vivo and spheroid colony formation in vitro) cells of these $3 \mathrm{GC}$ cell lines are more resistant to cisplatin than CD44- cells $[28,29]$. We observed that with the acquisition of cisplatin resistance, SGC7901 cells transitioned from epithelial state to mesenchymal state, the expression of the epithelial maker E-cadherin was downregulated, and the expression of mesenchymal makers $\mathrm{N}$-cadherin and Vimentin were significantly increased. At the same time, the bioinformatics analysis of the differentially-expressed genes showed that many genes were enriched in molecular mechanisms (using GO analysis), such as cell localization, locomotion, biological adhesion (biological process), cytoskeleton organization (cellular component), cell binding (molecular function), and pathways (using KEGG pathway analysis), such as cell adhesion molecules, ECM-receptor interaction, focal adhesion, PI3K-Akt signaling pathway, and Hippo signaling pathway, which, as mentioned above, are significantly correlated with EMT and CSCs properties [27,30,31]. These results show a strong mechanistic link of EMT, CSCs, and cisplatin resistance in SGC7901 cells.

Additionally, some of the bioinformatic results have been confirmed to be involved in cisplatin resistance in other cancers. For example, in the Rap1 signaling pathway, Lu Xiao and colleagues discovered that the cytoplasmic Rap1-NF-KB-BCL2 axis is a key mechanism in cisplatin resistance in non-small cell lung cancer [32]. Studies demonstrated that cisplatin-induced oxidative stress is one of the molecular mechanisms of cisplatin pharmacology [33], and our bioinformatic results showed that antioxidant activity might play a role during the acquisition of cisplatin resistance. There may be many underlying mechanisms that give rise to cisplatin resistance, because some of the GO and KEGG terms proposed in our study, such as metabolic process, immune system, and estrogen signaling pathway, are rarely reported. 


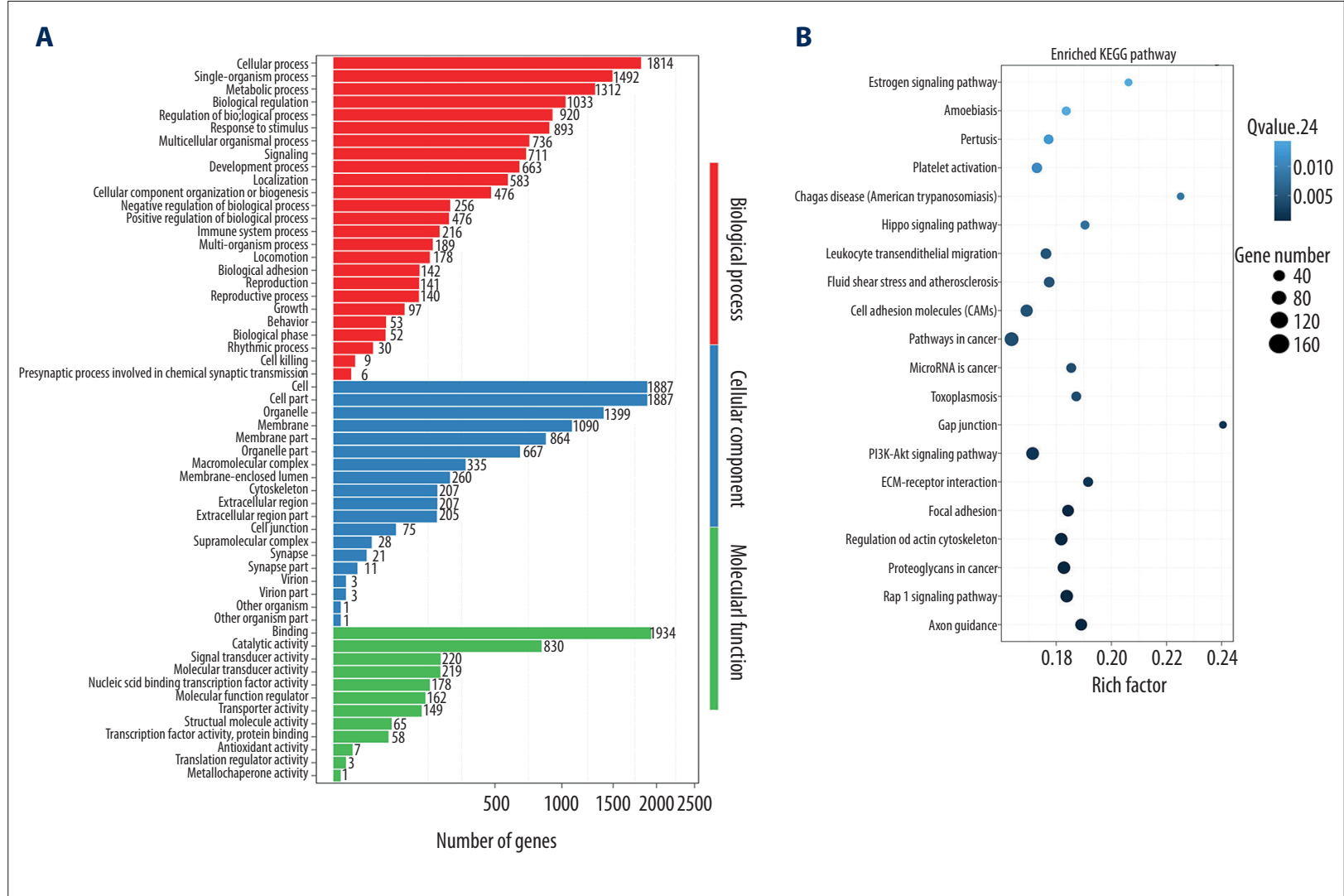

Figure 5. Bioinformatics analysis of the differentially expressed mRNAs. (A) GO analysis. (B) KEGG pathway enrichment analysis. The degree of color stands for the FDR q value; size of node stands for the gene numbers.

\section{Conclusions}

Taken together, the present study provides a global horizon of the function of the DEGs between SGC7901/DDP and SGC7901/S cells, and explores the potential mechanisms that induce cisplatin resistance. Although the past years have witnessed some great discoveries in cisplatin resistance of cancers, further investigation is still urgently needed.

\section{References:}

1. Chen W, Zheng R, Baade PD et al: Cancer statistics in China, 2015. Cancer J Clin, 2016; 66(2): 115-32

2. Torre LA, Bray F, Siegel RL et al: Global cancer statistics, 2012. Cancer J Clin, 2015; 65(2): 87-108

3. Chen W: Cancer statistics: Updated cancer burden in China. Chin J Cancer Res, 2015; 27(1): 1

4. Zong $L$, Abe $M$, Seto Y, Ji J: The challenge of screening for early gastric cancer in China. Lancet, 2016; 388(10060): 2606

5. Wohrer SS, Raderer M, Hejna M: Palliative chemotherapy for advanced gastric cancer. Ann Oncol, 2004; 15(11): 1585-95

6. Koizumi W, Narahara H, Hara T et al: S-1 plus cisplatin versus S-1 alone for first-line treatment of advanced gastric cancer (SPIRITS trial): A phase III trial. Lancet Oncol, 2008; 9(3): 215-21

7. Wagner $A D$, Grothe $W$, Behl $S$ et al: Chemotherapy for advanced gastric cancer. Cochrane Database Syst Rev, 2005; (2): CD004064

\section{Acknowledgments}

We thank the Editage Company for the language revision service.

\section{Conflict of interests}

None.

8. Sangrajrang S, Fellous A: Taxol resistance. Chemotherapy, 2000; 46(5): 327-34

9. Orr GA, Verdier-Pinard P, McDaid H, Horwitz SB: Mechanisms of Taxol resistance related to microtubules. Oncogene, 2003; 22(47): 7280-95

10. Lu C, Shan Z, Li C, Yang L: MiR-129 regulates cisplatin-resistance in human gastric cancer cells by targeting P-gp. Biomed Pharmacother, 2017; 86: 450-56

11. Pasello M, Michelacci F, Scionti I et al: Overcoming glutathione S-transferase P1-related cisplatin resistance in osteosarcoma. Cancer Res, 2008; 68(16) 6661-68

12. Liao JK, Zhou B, Zhuang XM et al: Cancer-associated fi broblasts confer cisplatin resistance of tongue cancer via autophagy activation. Biomed Pharmacother, 2018; 97: 1341-48

13. Crul M, Schellens JH, Beijnen JH, Maliepaard $M$ : Cisplatin resistance and DNA repair. Cancer Treat Rev, 1997; 23(5-6): 341-66 
14. Fang Z, Zhao J, Xie $W$ et al: LncRNA UCA1 promotes proliferation and cisplatin resistance of oral squamous cell carcinoma by sunppressing miR-184 expression. Cancer Med, 2017; 6(12): 2897-908

15. Fang Z, Chen W, Yuan Z et al: LncRNA-MALAT1 contributes to the cisplatinresistance of lung cancer by upregulating MRP1 and MDR1 via STAT3 activation. Biomed Pharmacother, 2018; 101: 536-42

16. Rabik CA, Dolan ME: Molecular mechanisms of resistance and toxicity associated with platinating agents. Cancer Treat Rev, 2007; 33(1): 9-23

17. Kalayda GV, Wagner $\mathrm{CH}$, Jaehde U: Relevance of copper transporter 1 for cisplatin resistance in human ovarian carcinoma cells. J Inorg Biochem, 2012; 116: 1-10

18. Xie XQ, Zhao QH, Wang H, Gu KS: Dysregulation of mRNA profile in cisplatin-resistant gastric cancer cell line SGC7901. World J Gastroenterol, 2017; 23(7): 1189-202

19. Schmittgen TD, Livak KJ: Analyzing real-time PCR data by the comparative C(T) method. Nat Protoc, 2008; 3(6): 1101-8

20. Orditura M, Galizia G, Sforza V et al: Treatment of gastric cancer. World J Gastroenterol, 2014; 355(7): 729-35

21. Cohen SM, Lippard SJ: Cisplatin: From DNA damage to cancer chemotherapy. Prog Nucleic Acid Res Mol Biol, 2001; 67(67): 93-130

22. And ERJ, Lippard SJ: Structure, recognition, and processing of cisplatin DNA adducts. Chem Rev, 1999; 99(99): 2467-98

23. Vitale I, Galluzzi L, Castedo M, Kroemer G: Mitotic catastrophe: A mechanism for avoiding genomic instability. Nat Rev Mol Cell Biol, 2011; 12(6): 385-92
24. Januchowski R, Zawierucha P, Andrzejewska $M$ et al: Microarray-based detection and expression analysis of ABC and SLC transporters in drug-resistant ovarian cancer cell lines. Biomed Pharmacother, 2013; 67(3): 240-45

25. Zhang Y, Zhang Q, Cao Z et al: HOXD3 plays a critical role in breast cancer stemness and drug resistance. Cell Physiol Biochem, 2018; 46(4): 1737-47

26. Cai XZ, Zeng WQ, Xiang $Y$ et al: iTRAQ Based quantitative proteomic analysis of nasopharyngeal carcinoma. J Cell Biochem, 2015; 116(7): 1431-41

27. Shibue T, Weinberg RA: EMT, CSCs, and drug resistance: The mechanistic link and clinical implications. Nat Rev Clin Oncol, 2017; 14(10): 611-29

28. Yoon C, Park DJ, Schmidt B et al: CD44 expression denotes a subpopulation of gastric cancer cells in which Hedgehog signaling promotes chemotherapy resistance. Clin Cancer Res, 2014; 20(15): 3974-88

29. Takaishi S, Okumura T, Tu S et al: Identification of gastric cancer stem cells using the cell surface marker CD44. Stem Cells, 2010; 27(5): 1006-20

30. Xia $P, X u X Y: P I 3 K / A k t / m T O R$ signaling pathway in cancer stem cells: from basic research to clinical application. Am J Cancer Res, 2015; 5(5): 1602-9

31. Hao J, Zhang $Y$, Jing $D$ et al: Role of Hippo signaling in cancer stem cells. J Cell Physiol, 2013; 229(3): 266-70

32. Lu X, Lan X, Shi X et al: Cytoplasmic RAP1 mediates cisplatin resistance of non-small cell lung cancer. Cell Death Dis, 2017; 8(5): e2803

33. Dasari S, Tchounwou PB: Cisplatin in cancer therapy: Molecular mechanisms of action. Eur J Pharmacol, 2014; 740: 364-78 